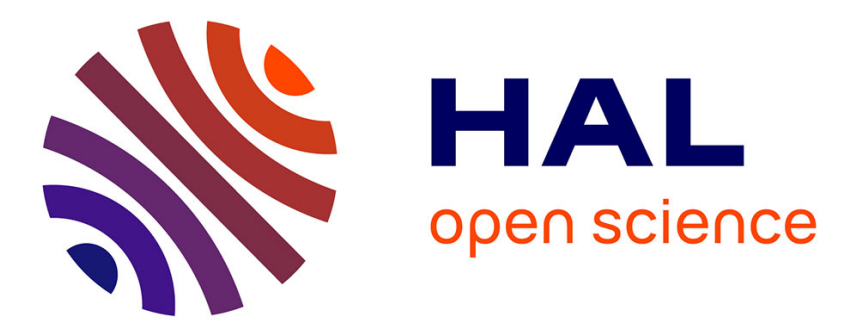

\title{
First characterization of two phase phenomena occurring during a rapid energy discharge in saturated carbon dioxide
}

Jean Muller, Romuald Rulliere, Adrien Abbate, Pierre Ruyer, Marc Clausse

\section{To cite this version:}

Jean Muller, Romuald Rulliere, Adrien Abbate, Pierre Ruyer, Marc Clausse. First characterization of two phase phenomena occurring during a rapid energy discharge in saturated carbon dioxide. Experimental Thermal and Fluid Science, 2021, 129, pp.110471. 10.1016/j.expthermflusci.2021.110471. hal-03381738

\section{HAL Id: hal-03381738 \\ https://hal.science/hal-03381738}

Submitted on 17 Oct 2021

HAL is a multi-disciplinary open access archive for the deposit and dissemination of scientific research documents, whether they are published or not. The documents may come from teaching and research institutions in France or abroad, or from public or private research centers.
L'archive ouverte pluridisciplinaire HAL, est destinée au dépôt et à la diffusion de documents scientifiques de niveau recherche, publiés ou non, émanant des établissements d'enseignement et de recherche français ou étrangers, des laboratoires publics ou privés.

\section{(ㅇ)(1) $\$$}

Distributed under a Creative Commons Attribution - NonCommercial - NoDerivatives| 4.0 


\title{
First characterization of two phase phenomena occurring during a rapid energy discharge in saturated carbon dioxide
}

\author{
Jean Muller ${ }^{\mathrm{a}, \mathrm{b}}$, Romuald Rulliere ${ }^{\mathrm{b}}$, Adrien Abbate $^{\mathrm{a}, \mathrm{b}}$, Pierre Ruyer $^{\mathrm{a}}$, Marc \\ Clausse $^{b}$ \\ ${ }^{a}$ Institut de Radioprotection et de Sûreté Nucléaire (IRSN), PSN-RES/SEMIA/LSMA, \\ BP3, St Paul-Lez-Durance 13115, France \\ ${ }^{b}$ Univ. Lyon, CNRS, INSA Lyon, CETHIL, UMR5008, Villeurbanne, F-69621, France, \\ Université Lyon 1, F-69622, France, romuald.rulliere@insa-lyon.fr
}

\begin{abstract}
The aim of this paper is to characterize two-phase phenomena occurring for a rapid energy discharge in the fluid leading to explosive vapour expansion. This study was motivated by the lack of macro-scale experiments characterizing those transient phenomena at high reduced pressure. For that purpose, a complete test section was designed based on the Joule effect to deliver the energy discharge. $\mathrm{CO}_{2}$ was chosen as working fluid, allowing to work at saturation and under saturation conditions.

Equipped with pressure sensors and a high-speed camera, the complete process is recorded during few seconds. The thermal shock in the carbon dioxide creates transient pressure peaks and sudden vapour production. The first observed pressure wave is well described as acoustics. This first wave is followed by a quick $(60 \mathrm{~ms})$ generation of vapour. The maximum volume of vapour produced is extracted from pressure fluctuations and matches the theoretical value. Following their creation, the bubbles flow upward in the test section as bubbly flow. Visual observation allows the characterization of the shape and the velocity of pertinent bubbles as part of a wobbling flow.

This project, motivated by the so-called Fuel Coolant Interaction (FCI) nuclear safety related problematic, brings consistent data allowing to better characterize the small scale processes for such transient vaporization phenomena.
\end{abstract}

This paper focuses on a single test performed under saturated conditions. 
Keywords: Boiling, Pressure peak, Experiment, Phase Change, Joule effect 


\section{Nomenclature}

\section{Acronyms}

BLEVE Boiling Liquid Expanding Vapour Explosion

FCI Fuel coolant interaction

\section{Greek symbols}

$\gamma \quad$ Laplace's coefficient

$\mu \quad$ Dynamic viscosity (Pa.s)

$\rho \quad$ Density $\left(\mathrm{kg} \cdot \mathrm{m}^{-3}\right)$

$\sigma \quad$ Surface tension $\left(\right.$ N.m $\left.{ }^{-1}\right)$

$\tau \quad$ Time scale (s)

\section{Latin symbols}

$\mathcal{L} \quad$ Specific latent Heat $\left({\left.\mathrm{J} . \mathrm{kg}^{-1}\right)}^{-1}\right.$

$\mathcal{T} \quad$ Wave power attenuation coefficient

a Minor axis length (m)

$b \quad$ Major axis length (m)

c Wave velocity $\left(\mathrm{m} . \mathrm{s}^{-1}\right)$

$C_{p} \quad$ Specific heat capacity $\left(\mathrm{J} . \mathrm{K}^{-1} \cdot \mathrm{kg}^{-1}\right)$

$C_{\text {cond }}$ capacitance (F)

D Diameter (m)

Eo Eötvös number

$f \quad$ Frequency $(H z)$

$g \quad$ Universal constant of gravitation $\left(9.81 \mathrm{~m} \cdot \mathrm{s}^{-2}\right)$

$h \quad$ Heat transfer coefficient $\left(\mathrm{W} . \mathrm{K}^{-1} \cdot \mathrm{m}^{-2}\right)$ 
$J \quad$ Calculus constant

$L \quad$ Length (m)

$M \quad$ Molar mass $\left(\mathrm{kg} \cdot \mathrm{mol}^{-1}\right)$

$m \quad$ Mass $(\mathrm{kg})$

Mo Morton number

$P \quad$ Pressure (Pa)

Re Reynolds number

$S \quad$ Surface $\left(\mathrm{m}^{2}\right)$

$T \quad$ Temperature $\left(\mathrm{K},{ }^{\circ} \mathrm{C}\right)$

$t \quad$ Time (s)

U Voltage (V)

$u \quad$ Velocity $\left(\mathrm{m} . \mathrm{s}^{-1}\right)$

$V \quad$ Volume $\left(\mathrm{m}^{3}\right)$

$v \quad$ Specific volume $\left(\mathrm{m}^{3} \cdot \mathrm{kg}^{-1}\right)$

z Gas constant

\section{Subscript}

b Bubble

bl Buffer layer

e Equivalent

exp Experimental

FS Full scale

$l \quad$ Liquid phase

meas Measured - i.e. experimental value 
$t \quad$ Energy deposit section

th Theoretical

$v \quad$ Vapour phase 


\section{Introduction}

Phase transition from the liquid phase to vapor is associated with significant transfer momentum due to volumetric expansion of the fluid. Therefore, one of the questions raised by the onset of boiling process is related not only to heat and mass transfer but also to potential scale of momentum transfers. Known situations where boiling process leads to the explosive expansion are Boiling Liquid Expanding Vapour Explosion -BLEVE- ([1], [2]), underwater explosion ([3], [4]) or vapour explosion [5]. In all these situations, high nonequilibrium conditions initiate the boiling process leading to both pressure pulse and high thermal to mechanical energy transfer. In the specific case of nuclear safety-related situations, vapour explosions have been widely studied for the estimation of the so-called "steam explosion induced containment failure risk", [5]. The corresponding accident scenario considers a large amount of high temperature liquid mixing with water. Another partially similar situation is associated with the potential consequence of fuel rod failure following reactivity accidental transient. During this typical accident, hot nuclear particle can be ejected from the rod into the coolant. This implies a sudden contact between hot solid particles and cold liquid ( $\Delta T$ around $2300 \mathrm{~K}$ ). It is the so-called Fuel Coolant Interaction (FCI). This instantaneous contact may generate destructive forces as presented in [6], [7] and [8].

To experimentally characterize the phenomena occurring during FCI different approaches were used in the aforementioned papers: fresh [6] or used [7], [8] nuclear rods were subjected to a deposit of energy until breakup. This breakup leads to the ejection, toward the coolant, of small nuclear particles (mean diameter near $15 \mu \mathrm{m}$ ) with high specific surface area (between 0.001 and $0.05 \mathrm{~m}^{2} / \mathrm{g}$ ) and high internal energy. It leads to significant fuel to coolant heat fluxes (around $10^{5} \mathrm{~W} / \mathrm{m}^{2}$ ). These energy discharges lead to high pressure transients (overpressure of a few $\mathrm{MPa}$ ) and intense vapour generation (a few litres of vapour generated in less than $1 \mathrm{~s}$ ). These experiments gave conversion ratio, which is the ratio between the generated mechanical energy (coolant motion related to fluid expansion through vaporization process) and the total energy deposition, between 0.1 and $1 \%$.

However, these works were performed with quiescent water at ambient pressure and temperature conditions, which are far from the nuclear power plant conditions. In a Pressurized Water Reactor (PWR), the coolant flows at high pressure and temperature, typically 155 bars and $300{ }^{\circ} \mathrm{C}$. These differences could have a major impact on the observed phenomena. Indeed, 
at high reduced pressure, the specific latent heat and the ratio between the density of liquid and vapour of water are significantly different from the ones at ambient conditions.

To deeply understand FCI, a fine characterization of the vaporization dynamic during fast heating (less than $10 \mathrm{~ms}$ ) is needed. Pressure transients for thermal shock at micro scale were characterized in different experimental works. These tests were respectively performed with a Pt wire $(10 \mu \mathrm{m}$ of diameter and $1 \mathrm{~mm}$ long for $120 \mu \mathrm{J}$ of deposit [9]) and a thin film microheater $(100 \mu \mathrm{m}$ long and $100 \mu \mathrm{m}$ large allowing $60 \mu \mathrm{J}$ of deposit [10]). The related papers outline different phases leading to a vapour explosion in still water at atmospheric conditions. A strong correlation is outlined between the pressure transient and the vapour generation for several energy deposit in the fluid.

The herein above described papers mainly depict phenomena at small reduced pressure. Hence, a dedicated experiment is needed to study vapour explosion phenomena under various sub-cooling conditions and energy discharges. This should permits to fully characterize the phenomena occurring during FCI and to validate a computational code developed at IRSN. For this purpose, a model fluid was selected and a specific test bench developed and built (EDITE for Etude d'un Dépôt Important et Transitoire d'Energie). They are both presented in the first section. The second and third section deal with a limited amount of data which bring extensive information on rapid boiling phenomena.

This paper focuses on a single test performed under saturated conditions.

\section{Experimental setup}

The experimental device has been designed to rapidly transfer heat to pressurized liquid and characterize the subsequent transient boiling process and flow dynamic, [11]. The experimental device is made of four systems detailed on figure 1:

- The fluid test section - a watertight tank contains the heating element, the liquid and a gaseous buffer layer;

- The heating system - an electrical loop delivers power to the heating element thanks to the Joule effect;

- The thermodynamic conditions control system - a system of liquid and gas tanks fills the fluid test section with pressurized fluids. A climatic chamber encloses the test section to control temperature; 
- The acquisition system - several sensors and visualization equipment.

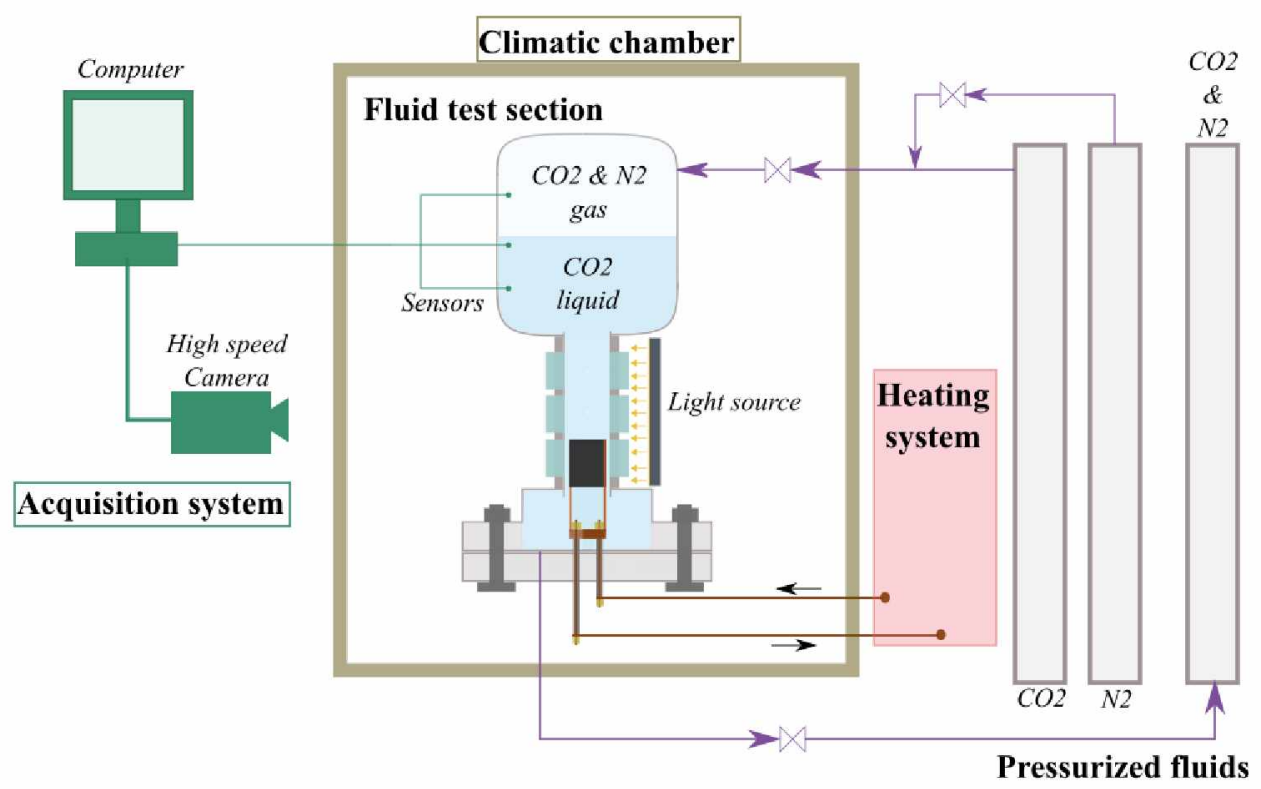

Figure 1: Complete experimental setup

\subsection{Fluid selection}

The FCI phenomenology has been observed in some experimental conditions that remain far from the reactor conditions in terms of water temperature and pressure, the latter determining some key properties for boiling processes (e.g. latent heat, liquid-vapor density ratio, heat conductivity and capacities, surface tension). Due to experimental constraints, it is not possible to reproduce the phenomenon in those high pressure/high temperature conditions. The experimental constraints mainly concerns safety and cost issues. It is far from the actual goal to fully scale the experiments in order to simulate the corresponding accidental conditions, but scaling of the bench has been made to approach some key features of those conditions. The goal of this paper is to show how the actual choices for the experimental design allow to reproduce the phenomenology. Further study will then consider how the intensity of the events (pressure peak, fluid expansion) actually scales with 
experimental conditions (energy, pressure, temperature). Therefore only a short summary of the principles that guided the design of the experiments is presented in this paper.

$\mathrm{CO}_{2}$ has been chosen since it allows studying a high reduced pressure fluid at convenient lab temperature and pressure: this allows reproducing the liquid-vapor density ratio of the accidental situation. The amount of energy released in the fluid corresponds to the specific heat of the fuel that has been scaled for the experiments with respect to the latent heat of the fluid surrounding the heating element. The scaling of this heating element has been made thanks to kinetics considerations. Mechanical relaxation time of the surrounding fluid $\left(\tau_{\text {mech }}\right)$ is scaled by the typical size of the device $\left(L_{c}\right)$ over the compressibility of the surrounding fluid as $\tau_{\text {mech }}=L / c_{s}$. For the boiling process to occur over relatively comparable time scales, energy has to be

1. rapidly deposited by the electrical circuit to the heating element. This constraint defined the choice for the capacitors of the electrical system - see section 2.4

2. rapidly transferred to the fluid, the latter thermal time scale say $\tau_{\text {th }}$ there being evaluated from a basic energy balance of the heating element considering a typical boiling heat transfer coefficient.

The size and shape (volume over surface ratio) of the heating element have been chosen to get the ratio $\tau_{\text {th }} / \tau_{\text {mech }}$ as low as reasonably possible; this time ratio is still large with respect to the one we could estimate from the accidental conditions.

For the studied test, the pressure was set to 29 bars. This working pressure is far from equivalent reactor conditions (50 bars). It leads to a density ratio $\left(\rho_{l} / \rho_{v}\right) 2$ times higher than the one observed in reactor conditions and implies a more abundant vapour creation and higher pressure transients. This first experiment at lower pressure is relevant to outline most of the phenomena expected which are more difficult to distinguish at higher reduced pressure. The design of the device was chosen to sustain higher pressure conditions that will be studied in future tests.

\subsection{Fluid test section}

The fluid test section, presented on figure 1, is composed of two main parts: 
- a narrow cylindrical tube filled with liquid. It contains the heating element in its lower region. This part is called the Energy deposit section

- a cylindrical vessel mounted the Energy deposit section. It is partially filled with liquid resulting in a gaseous buffer layer at its top. This part is called the Buffer vessel

Both parts are independently presented in this subsection.

Energy deposit section. The inside diameter of the narrow tube of stainless steel is $D_{t}=25.6 \mathrm{~mm}$ and its length is $40 \mathrm{~cm}$. The heating element is made of three parallel plates of tungsten, which was chosen for its relatively high melting temperature $(3500 \mathrm{~K})$, allowing the heating element to endure high energy deposits. It has been designed to have relatively low thermal mass, high electrical resistivity and large areas to maximize the heat transfer toward $\mathrm{CO}_{2}$. Figure 2 shows the $3 \mathrm{D}$ drawing and a picture of the heating element.Each tungsten plate is $60 \mathrm{~mm}$ high, $21 \mathrm{~mm}$ wide and $0.4 \mathrm{~mm}$ thick. They are attached to each other with brass screws. The plates are connected to the electrical loop with successive copper elements of larger cross section.
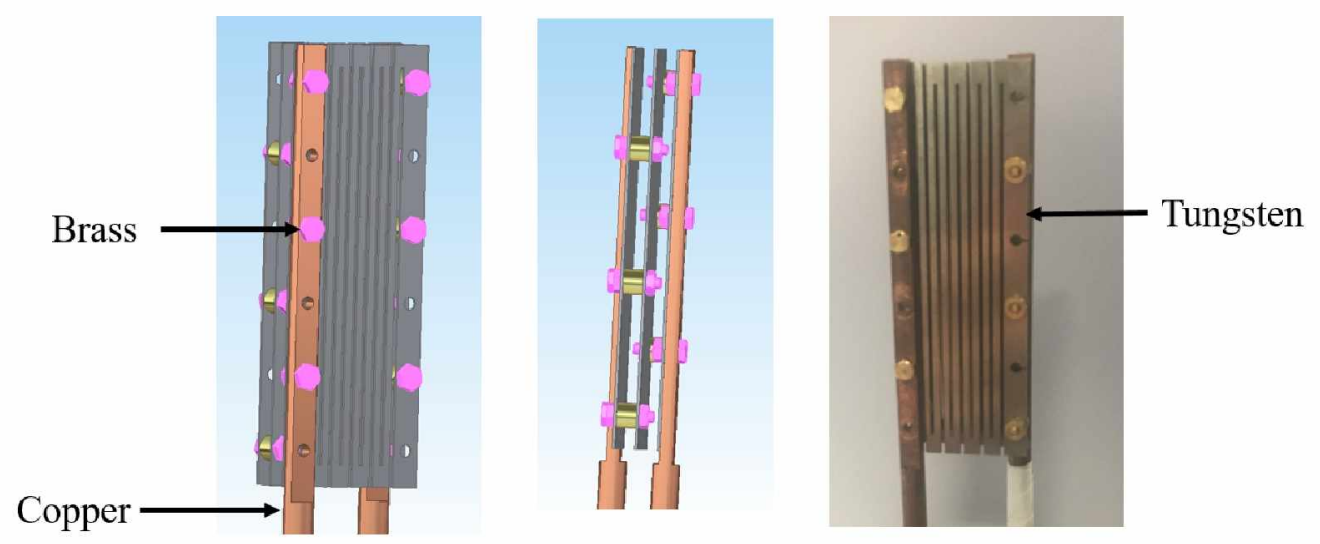

Figure 2: Heating element. Tungsten plates are assembled with brass elements (in pink and gold). The copper connectors are represented in orange.

Dense copper bars are connected to the electrical heating system with protected copper bars (busbars). Boiling and flow dynamics could be observed by ombroscopy thanks to three vertically arranged facing viewports $(\mathrm{D}=16.5 \mathrm{~mm})$ made of Borosilicate glass. As it can be seen on the figure 3-(c), 
the heating element can be seen through viewport V1, allowing to observe boiling onset. Bubble rising can be observed through V2 and V3. Two dynamic pressure sensors (P1 and P2) are located at V2 and V3 levels (figure 3 -(a)) to record differential pressure variations in the tube.

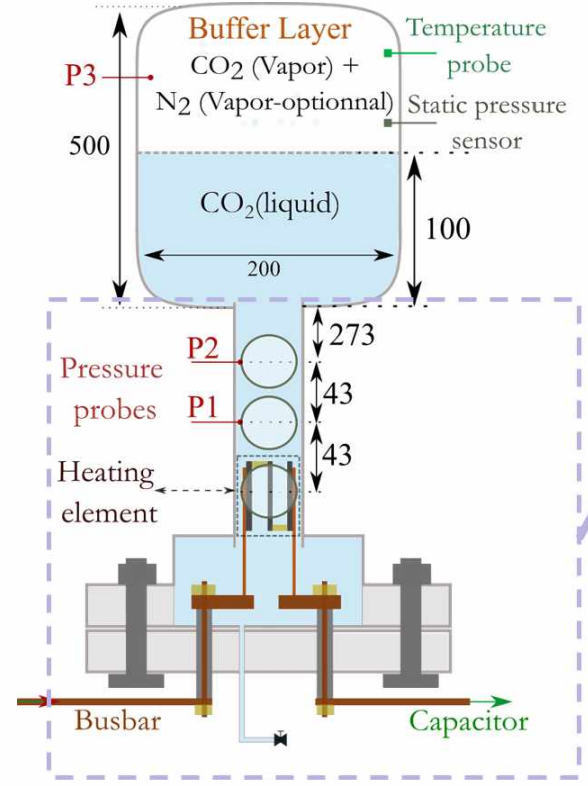

(a)

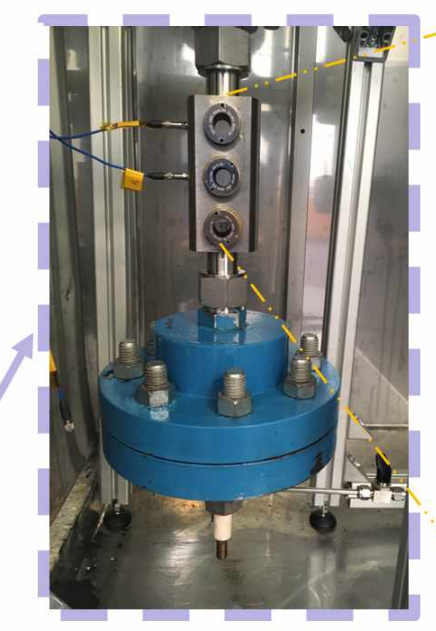

(b)

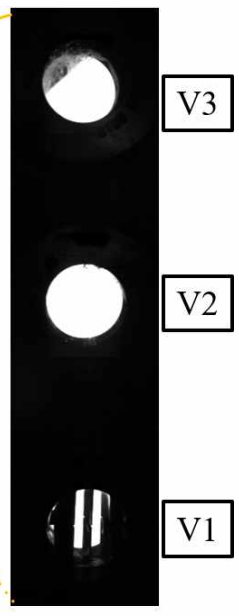

(c)

Figure 3: Fluid test section. (a) Drawing of the complete test section (heat deposit section and buffer tank) and the sensors with their position. (b) Picture of the heat deposit section. (c) Frame of the visualization zone obtained with the high speed camera

Buffer vessel. The buffer vessel ( $D=200 \mathrm{~mm}, V=13 \mathrm{~L})$ is a reservoir of heat capacity. It is at a relatively far distance from the heating element and also acts as an expansion tank thanks to its buffer layer. Temperature and static pressure sensors allow for monitoring the initial thermodynamic conditions of the tests. The dynamic pressure sensor P3 records the compression of the buffer layer.

\subsection{Thermodynamic conditions control systems}

The upper part of the fluid test section vessel is connected to both pressurized nitrogen and carbon dioxide reservoirs (see figure 1). This system allows controlling the liquid $\mathrm{CO} 2$ level within the vessel as well as the partial 
pressure of nitrogen in the buffer layer independently. The whole fluid test section is enclosed in a climatic chamber (Liebherr BGPv 8420) to control the temperature.

As a consequence, the initial thermodynamic state within the fluid test section can be set over a range of initial conditions, from saturated liquid $\mathrm{CO}_{2}$ to subcooled liquid $\mathrm{CO}_{2}$. After filling the test section with liquid carbon dioxide, the addition of gaseous nitrogen in the buffer layer compresses the carbon dioxide. The add of uncondensable gas set the thermodynamic state of the liquid $\mathrm{CO}_{2}$.

In this paper, a single test condition at saturation is discussed.

\subsection{Electrical heating system}

In order to achieve a fast heating of the tungsten plates by Joule effect, a transient electrical discharge of capacitors is considered. The loop has been designed to produce a discharge of $1.4 \mathrm{~kJ}$ in $3 \mathrm{~ms}$. The current is generated by a rack of nine film capacitors of $3 \mathrm{mF}$ each, giving a total capacitance of $C_{\text {cond }}=27 \mathrm{mF}$. The discharge time is controlled by phase control thyristor (T1500N).

The coated copper plane bars named busbars on Figure 3 are connected to the heating element with successive dense copper bars.

\subsection{Acquisition system}

Initial thermodynamic conditions. The initial thermodynamic conditions in the test section and in the climatic chamber are monitored. Static pressure is measured with an Optibar P1010C from Krohne. Its measurement interval is $P \in[0.1$ bar; 100 bar $]$ with an uncertainty of \pm 0.25 bar. Temperature in the buffer vessel is measured thanks to a plunging thermocouple. It is a B-class probe made under the norm NF EN 60751. For the volume of liquid in the test section, a bypass level indicator (BM 26A from Khrone) is used. Its uncertainty is $10 \mathrm{~mm}$ which represents $V_{\mathrm{CO}_{2}}=V\left[\mathrm{~m}^{3}\right] \pm 710^{-4} \mathrm{~m}^{3}$. This represents a $5 \%$ uncertainty on the volume of gas in the buffer layer. All the sensors (except the Bypass level indicator) are represented in Figure 3.

High-speed visualization. A high-speed camera (Photron Fastcam 120k) is positioned outside the climatic chamber, and focused on the three viewports area through a window placed on the chamber wall. Typical frame is reproduced on figure 3-(c). A square white LED blacklight device (Phlow SLLUB white led Blacklight 100x100, luminance: $53000 \mathrm{~cd} . \mathrm{m}^{2}$, uniformity: $98.77 \%$ ) 
illuminates the imaging area through the opposite viewports. The Photron Fastcam Viewer (PFV) software controls the high-speed camera. The following settings were chosen to record videos: image size was set to 768 pixels*256 pixels. It gives a spatial resolution within the test section of approximately $5.8 \mathrm{px} / \mathrm{mm}$. The frame rate was set to 5000 frames per second. This generates a $1.3 \mathrm{~s}$ long video of the test starting from the beginning of the electrical discharge.

Dynamic pressure. The phenomena occurring during the transient heat deposit have a time scale smaller than $1 \mathrm{~ms}$. A comprehensive overpressure measurement was then designed to capture these small time scales. Three piezoelectric sensors (PCB 113B21) connected to a PCB signal conditioner are used at different heights in the test section. These sensors are named P1, P2 and P3 on figure 3 and measure dynamic pressure. Which mean, that they only record the pressure fluctuations from an initial state. The sensor precision is $7 \mathrm{~Pa}$. For the uncertainty, using two test procedures (AT601-11 and AT601-6), the overpressure measurement has an uncertainty equal to $1.3 \%$. Furthermore, the non-linearity response of the sensors is $0.4 \% \mathrm{FS}=5.5 \mathrm{kPa}$ (FS meaning Full-Scale).

Energy deposited in the heating element. Power induced by Joule effect across the tungsten plates of the heating element is calculated from the intensity and voltage measurements respectively with LEM LF2010-S (resolution of $200 \mathrm{~mA}$ ) and LEM CV 3-500 sensors (resolution of 1.2 V). Current measurement overall accuracy takes into account magnetic offset, electrical offset, sensitivity and linearity. It gives a $\pm 4 \mathrm{~A}$ uncertainty on the measured intensity. The voltage measurement accuracy $\left(0.2 \%\right.$ of $\left.U_{F S}\right)$ and offset $\left(U_{0}\right)$ gives a total accuracy of $\pm 0.7 \mathrm{~V}$. The uncertainty on the power calculations is $\pm 2 \%$.

Acquisition system. The dynamic acquisition is completed by two $9223 \mathrm{DAQ}$ modules (one for the overpressure measurement and one for the currentvoltage measurement) set to high frequency $(500 \mathrm{kHz})$. Moreover, the acquisition system was developed to synchronize the overpressure measurements with the high-speed visualization thanks to a specific trigger signal furnished by the electrical system.

A 9401 DAQ module is dedicated to the initial thermodynamic conditions records. Its sampling frequency is $2 \mathrm{~Hz}$. 


\section{Experimental results}

In this paper, a unique experiment is considered. It has been selected for the relevance of the phenomena observed. The following test conditions are considered. The fluid test section is partially filled with pure $\mathrm{CO}_{2}$. Initial conditions correspond to the saturation state $\left(P_{0}=2.96 \mathrm{MPa}, T_{0}=266.8 \mathrm{~K}\right)$ with a gaseous buffer layer of $12.1 \mathrm{~L}$. The initial voltage of the condensators is set to $70 \mathrm{~V}$, that corresponds to a $55 \mathrm{~J}$ discharge within the heating element.

\subsection{Pressure transients}

The experiment is triggered by the discharge of the capacitors in the heating element. Figure 4 shows the typical pressure wave obtained. The discharge is a first order system ( $\tau=3 \mathrm{~ms}$ ) resulting in a $90 \%$ of the energy deposit within the first $9 \mathrm{~ms}$. The applied electrical power reaches a maximum of $2.2410^{4} \mathrm{~W}$.
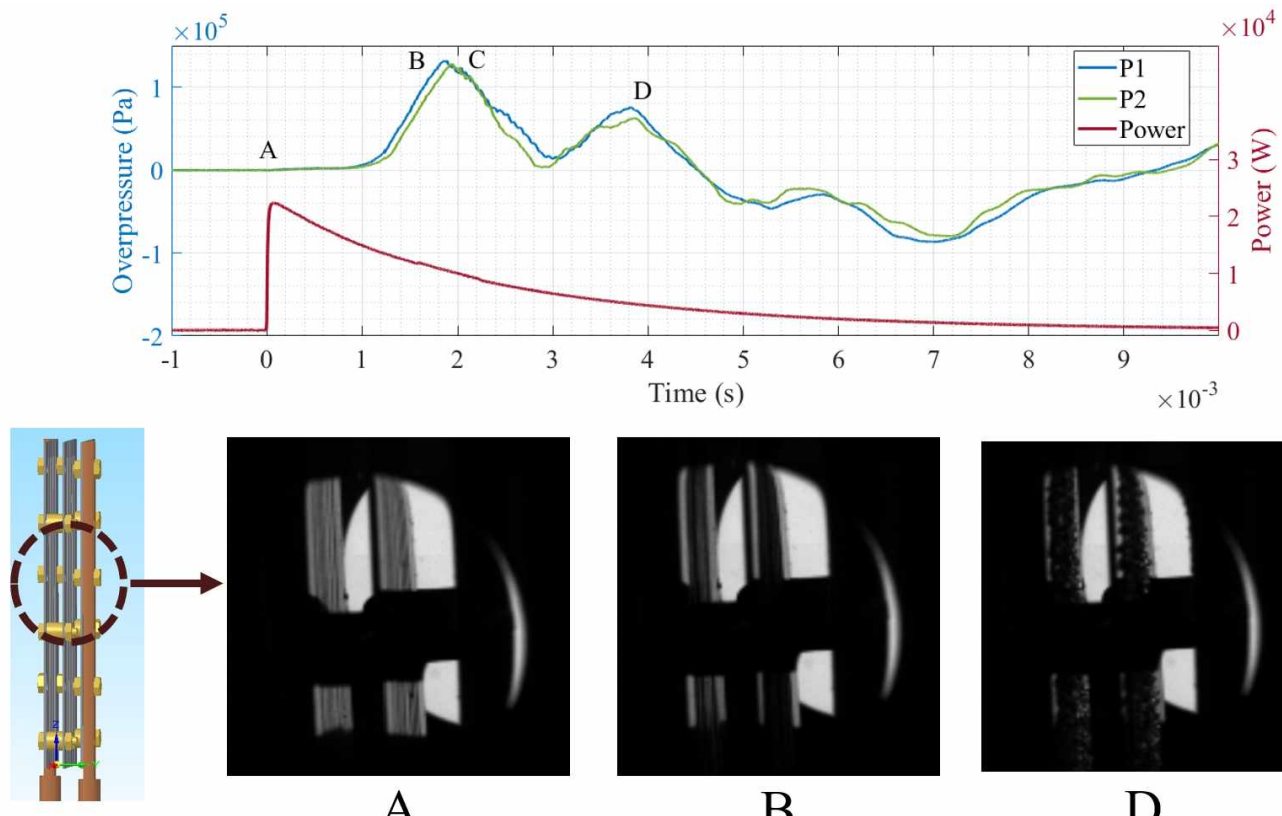

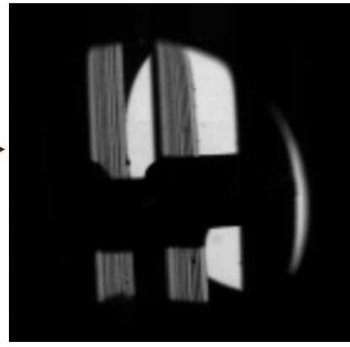

A

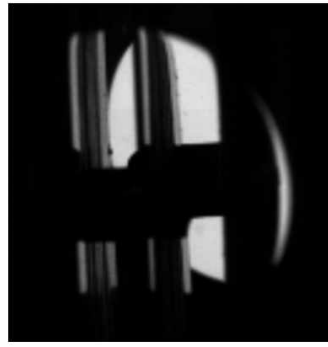

B

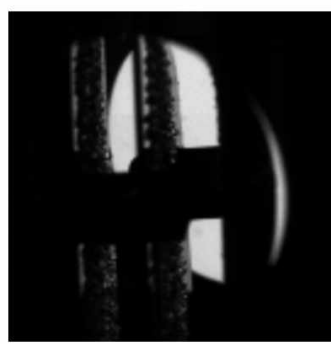

$\mathrm{D}$

Figure 4: Top part: Pressure transients (left y axis) recorded after the power discharge in the heating element (right $\mathrm{y}$ axis). Bottom part: frames captured during a similar

On the same figure, is shown the pressure wave produced by the transient deposit of energy. First at rest (A), the two dynamic pressure transducers (P1 
and $\mathrm{P} 2$ ) record a pressure wave characterized by a large positive overpressure pulse (B at P1 and $\mathrm{C}$ at P2) followed by damped oscillations around the initial 0 value. The delay between the point $\mathrm{B}$ and $\mathrm{C}$ is shown on zoomed view (green dashed rectangle). The first peak increase's rate (A to $\mathrm{B}$ ) is equal to $163 \mathrm{MPa} / \mathrm{s}$. This increase rate is of the same order of magnitude than the one observed in BLEVE, [1]. But it seems lower (factor 3) than the ones observed in vapour explosion [9]-[10].

Let us consider how the pressure transient is correlated with phase change dynamics over the wall. The experimental test has been repeated (same thermodynamics condition and same energy deposited ) with enhanced camera settings. The corresponding recorded frames are presented on figure 4. The screenshot on the left hand side of the figure outlines the orientation of the heating element. Before the deposit of energy (A), the tungsten sheets appear as grey. At the time of the first pressure peak (B), the heated part of the sheets are instantaneously covered by a black layer. Later on observation, instant D reveals that this black layer is a vapor blanket covering the wall: bubbles start growing from this layer at still relatively early times of the transient. Small volume of vapour appears on the tungsten sheets. These frames confirm that the first pressure peak is due to explosive phase change.

For such a rapid heating of the element, heterogeneous nucleation is not thought as being a limiting process. Since for rapid heating conditions, the nucleation conditions are still related to classical Hsu criterion corresponding to rather low wall superheat [12]: a condition that is reached very early with our transient heating of the wall starting from saturation conditions. The visual observation confirms that vapor is formed over the wall. Furthermore, no homogeneous nucleation phenomena were observed even when pressure dropped below the initial pressure level.

Under the first assumption of one dimension overpressure propagation, the pressure wave propagates vertically in the test section. Figure 5 shows the wave journey in correspondence with overpressure measurement on Figure 4. The delay between the events $\mathrm{B}$ and $\mathrm{C}$ could be calculated as $\Delta t_{(B / C)} \approx 82 \mu \mathrm{s}$. 


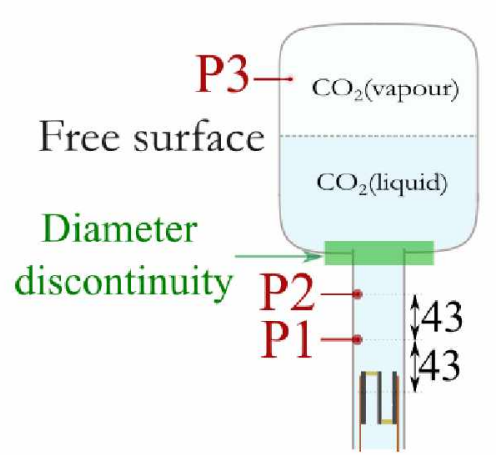

A

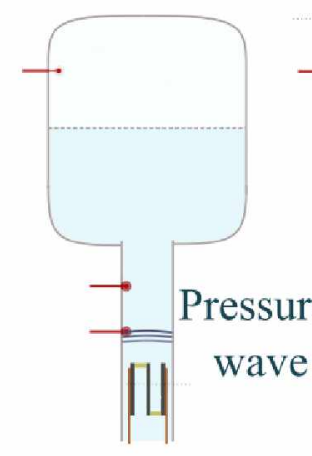

B

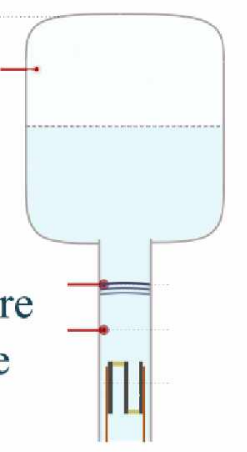

$\mathrm{C}$

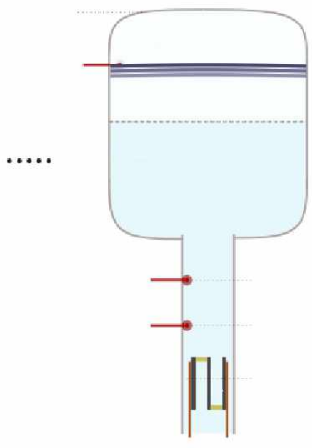

$\mathrm{D}$

Figure 5: Pressure wave propagation in the test section from A (at rest) to D (reaching the sensor P3). Only the first order pressure wave is considered (without wave bounces)

Assuming that the overpressure peak source is located at the heating element and that the pressure propagates as a plane wave along the tube, the delay should correspond to the distance between the sensors P1 and P2, $\Delta L_{P 1 / P 2}=4.310^{-2} \mathrm{~m}$, divided by the sound velocity in the liquid $\mathrm{CO}_{2}$. The evaluation of the sound velocity $c_{e x p}$ from $\mathrm{P} 1$ and $\mathrm{P} 2$ measurements leads to a slightly lower value with respect to the theoretical one $c_{t h}$ calculated at the test's temperature and pressure ([13]):

$$
c_{\text {exp }}=\frac{\Delta L_{P 1 / P 2}}{\Delta t_{P 1 / P 2}}=\frac{\Delta L_{P 1 / P 2}}{\Delta t_{(B / C)}}=524 \mathrm{~m} / \mathrm{s} \quad<c_{t h}=599 \mathrm{~m} / \mathrm{s}
$$

The experimental value is obtained by considering the distance between the center of each pressure transducer active zone. The uncertainty of this calculation due to geometric consideration (active zone position) is $\pm 70 \mathrm{~m} / \mathrm{s}$. The remaining difference between the experimental value and the theoretical one could be explained by the non purely planar wave propagation of the pressure peak across the tube and to the relatively large pressure variation amplitude (that goes beyond the classical small perturbation theory).

After the first peak, the overpressure first decreases steeply toward 0 , then increases till a second peak occurring at $t=0.004 \mathrm{~s}$, and then falls to values under the initial ones. Those oscillations could be associated to acoustics behaviours within the tube and wave reflections at the acoustic impedance variation locations. During the wave journey from the heating element to the top of the test section, the first change of acoustic impedance occurs at the diameter discontinuity (see Figure 5). The time scale of back and forth 
from the sensor P1 to the abrupt change of diameter is $0.8 \mathrm{~ms}$. This time scale matches well with the delay between the beginning of the increase of overpressure (few instants after A) and B, justifying the influence of wave return in the decrease of overpressure. Then, after the first peak, the shift between P1 and P2 signals corresponds no more to a constant delay between both sensors.

After the abrupt change of section, the pressure wave travels in the large vessel finally reaching the pressure transducer $\mathrm{P} 3$ at $t=3.8 \mathrm{~ms}$ ( $\mathrm{D}$ in figure 4 ). Figure 6 shows the comparison of the recorded overpressure P1 and P3. The steep increase of the P3 signal (D) clearly occurs with a delay with respect to P1 (B) and P2 (C). The time delay between these overpressure peaks is noted as $\Delta t_{(B / D)}$ and is equal to $1.9 \mathrm{~ms}$. This delay is comparable with the theoretical time of travel from $\mathrm{P} 1$ to $\mathrm{P} 3$ (noted $\Delta t_{P 1 / P 3}$ ), as follows :

$$
\Delta t_{P 1 / P 3}=\frac{L_{P 1 / f s}}{c_{t h}^{(l i q)}}+\frac{L_{f s / P 3}}{c_{t h}^{(\text {vap })}}=1.9 \mathrm{~ms}=\Delta t_{B / D}
$$

Where $c_{t h}^{(v a p)}$ is the velocity of the sound in carbon dioxide vapour whose equal to $215 \mathrm{~m} / \mathrm{s}([13])$ in these test conditions and $f s$ stands for free surface (between liquid $\mathrm{CO}_{2}$ and its vapour). $L_{P 1 / f s}$ and $L_{f s / P 3}$ are respectively equal to $416 \mathrm{~mm}$ and $250 \mathrm{~mm}$.

It shows a very good agreement with the actual peak recorded by $\mathrm{P} 3$ and therefore illustrates the importance of acoustic phenomena during the very first times of the test. However the order of magnitude of the over-pressure at the point $\mathrm{B}$ and $\mathrm{D}$ are rather different. The ratio between the maximum of P3 and the maximum of P1 is equal to 0.0013 .

The difference of order of magnitude of the overpressure between the point $\mathrm{B}$ and $\mathrm{D}$ could be explained by the abrupt change of diameter and the change of fluid. These singularities lead to a global transmission coefficient $(\mathcal{T})$ equal to 0.0019

The observed overpressure attenuation and the theoretical wave attenuation calculated are of the same order of magnitude. This justifies the different scales for P3 and P1 signals during the transient.

After the first acoustic peak, the sensor P3 exhibits a smaller (11 times less, see figure 6) and slower increase, from E to F. After F, the overpressure P3 stabilizes around a maximum of $550 \mathrm{~Pa}$. The signal P3 is fluctuating due to waves interference but does not show negative values during the first stages (from E to F). 


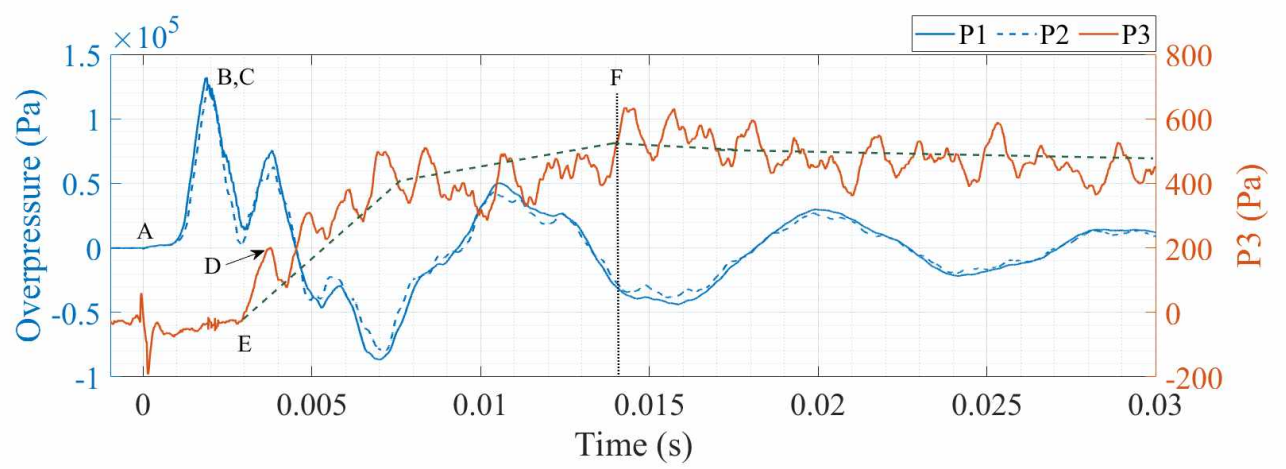

Figure 6: Overpressure with respect to time illustrating the peak delay between different sensor locations. A,B,C and D stands for the first acoustic pressure peak dynamic. E and F delimit the different phase exhibited by P3

\subsection{Visual observation of vapour/bubbles formation and flow}

Figure 7 shows selected frames recorded by the high-speed camera for the first $0.45 \mathrm{~s}$ of the test. It illustrates the different stages of the boiling process that occurs over the heating element. The boiling onset is difficult to determine precisely with the space resolution chosen. Very first boiling phenomena are discussed in section 4.1. Millimetric bubbles appear around $0.009 \mathrm{~s}$. This timing is fully consistent with the visual observation of boiling onset over the wall as illustrated in figure 6. During the next period, $t \in[0.009 \mathrm{~s} ; 0.049 \mathrm{~s}]$, the bubbles grow over the heating element and large voiding is observed through viewport V1. Bubbles departure can be seen after $0.049 \mathrm{~s}$. But individual bubbles are tough to distinguish. The bubbles then flow upward in the tube. First third of the bubbles reaches viewport V2 around $t=0.18 \mathrm{~s}$ and V3 around $t=0.45 \mathrm{~s}$. At this time, boiling over the heating element is no more observed, indicating that most of the heat has been transferred toward the fluid. Through V2, bubbles are relatively small and isolated while a relatively large bubble is observed at V3; this indicates bubbles coalescence within the tube. Due to the resolution, a direct comparison with the overpressure observations from the previous subsection is impossible. Nevertheless, numerous and distinct bubbles are observed, allowing geometric and dynamic characterization presented in section 4 . 


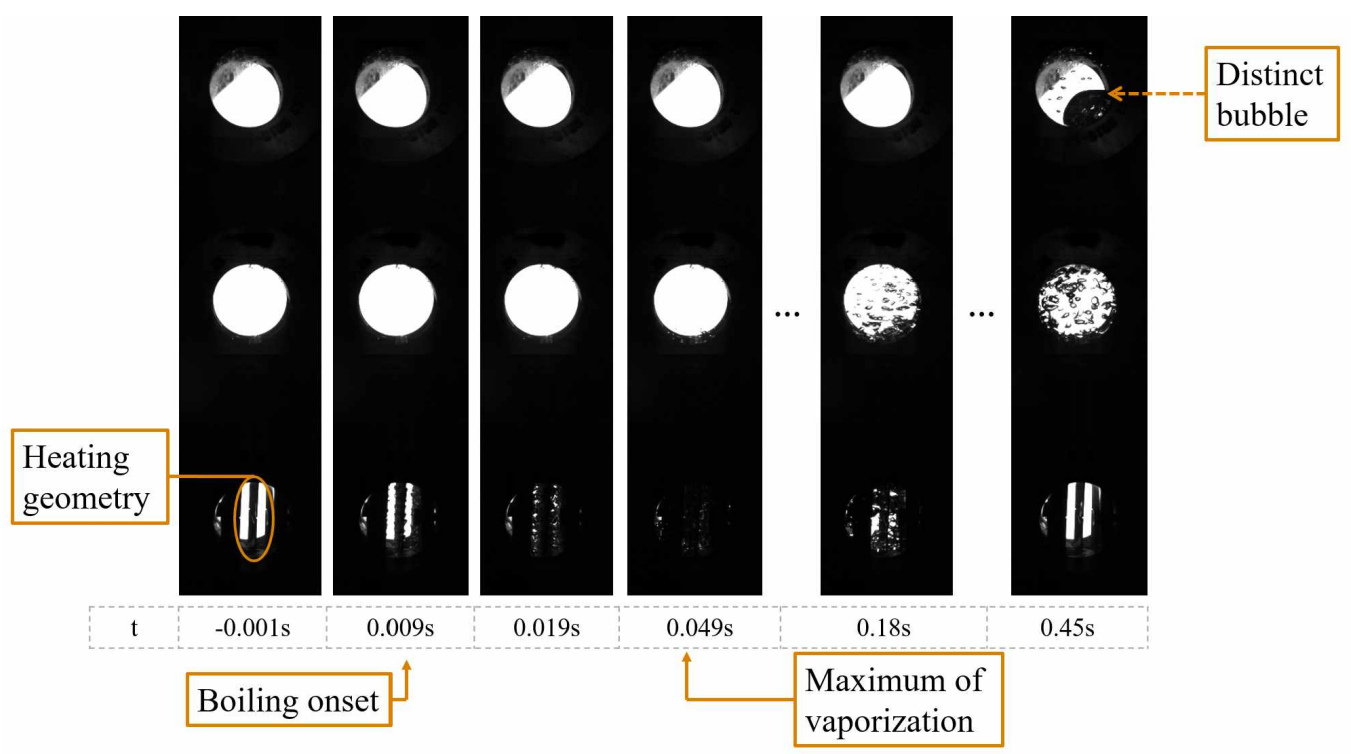

Figure 7: Summary of the different stages of the vaporization

\subsection{Compression and relaxation of the upper vapour layer}

Figure 8 shows the overpressure dynamics from maximum of overpressure (F-G) to the end of the test. After the increase of overpressure (E-F), the overpressure exhibits a small plateau (F-G) and then a slow (time scale of $1 \mathrm{~s}$ ) decrease (G-end). The increase of overpressure has to be related to the vapour generation near the heating element. By creating vapour at the bottom of the test section, the liquid (incompressible) is pushed upward and compress the buffer layer. This compression can be foreseen from the early times of the transient (even before 0.01 s) that would imply that vapour production occurs at the early stage of the test. Rather large oscillations are also observed, the period approximately equal to $0.04 \mathrm{~s}$. The decrease observed from point $\mathrm{G}$ is due to the chosen dynamic pressure sensor and its relaxation time. 


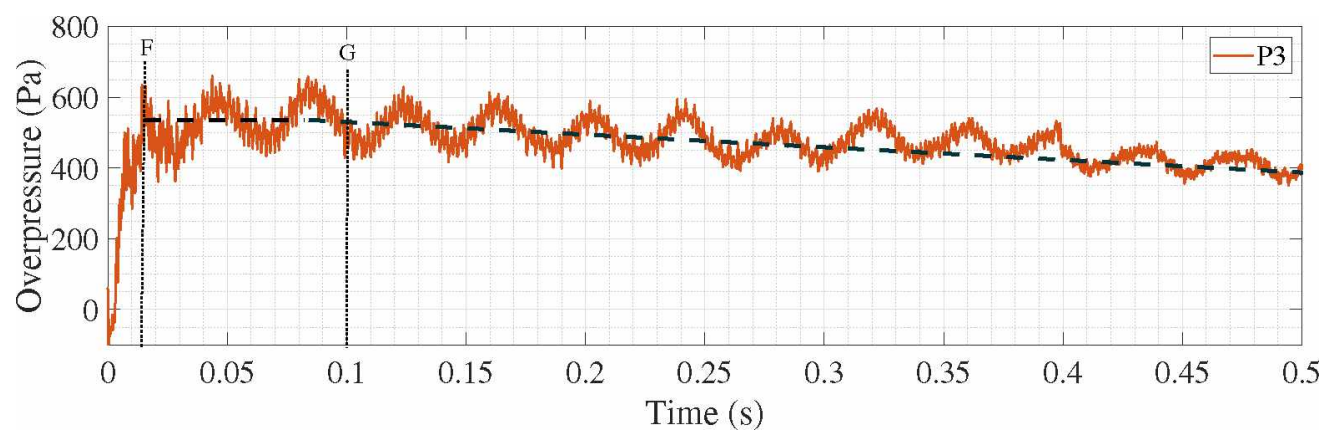

Figure 8: Overpressure measurements in the buffer layer after the transient deposit

\section{Result analysis and discussion}

\subsection{Onset of vaporization}

By zooming on the viewport V1, the first bubbles are observed at 1.9 ms. This very early boiling phenomenon corresponds to a time for which approximately $45 \%$ of the energy has been deposited. This appearance is directly followed by the growth of the bubbles. The growth is accompanied by a vertical movement of the vapour mass. Even though the bubbles appeared to be still attached to the surface during their growth, they clearly undergo a synchronized back and forth vertical movement. The figure 9 displays this kinetic. 


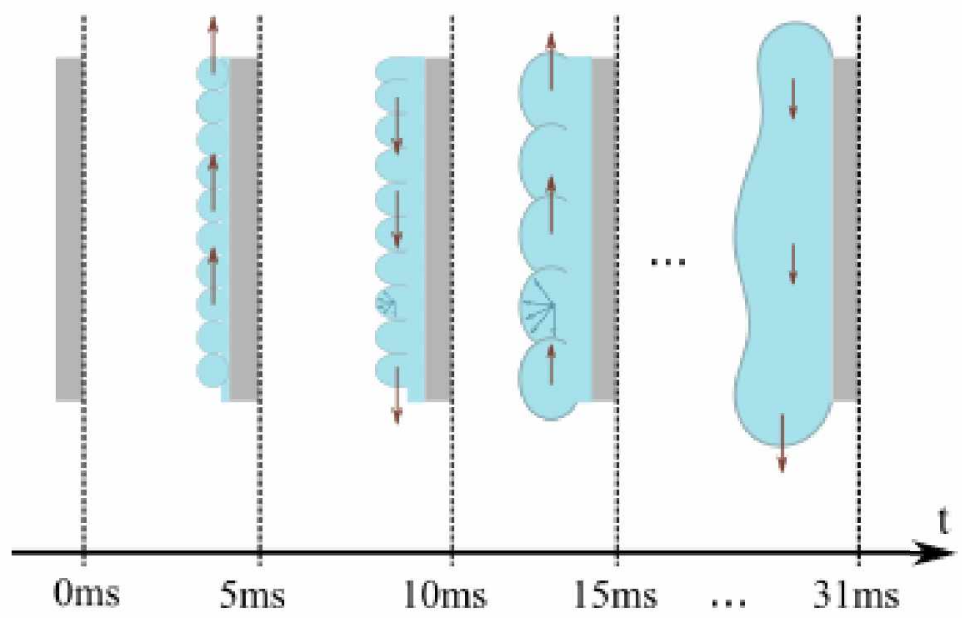

Figure 9: Bubble motion during the first milliseconds. A heating sheet is represented in grey and the vapour in blue. The growth of the bubbles is represented thanks to blue arrows. Their motion is schematized with red arrows.

At these early times of the boiling process, individual tiny bubbles can be distinguished. P.I.V (Particle Image Velocimetry) is used to determine their sliding velocity alongside the heating element.

The vertical overpressure gradient within the liquid is estimated from P1 and $\mathrm{P} 2$ measurements according to $\frac{\partial P}{\partial z} \simeq \frac{P 2-P 1}{L_{P 2 / P 1}}$. Figure 10 shows a comparison between the overpressure gradient and the vertical velocity magnitude of the bubbles (bottom). The velocity magnitude exhibits damped oscillations (so called back and forth previously) whose time scale is close to $9 \mathrm{~ms}$ and whose maximal amplitude (first oscillation) is approximately $0.7 \mathrm{~m} / \mathrm{s}$. It exhibits alternate positive and negative values which are related to the vertical velocity magnitude ones.

Comparison of the time variation for bubbles vertical velocity and liquid overpressure gradient within the tube clearly exhibits a strong correlation between both quantities. The extrema of the gradient corresponds to a zero value for the velocity. An increase of the overpressure gradient corresponds to positive velocity (the bubbles ascend) and inversely. 



Figure 10: Velocity of the bubble on the tungsten compared with the overpressure difference $\left(P_{2}-P_{1}\right)$

This velocity magnitude to overpressure gradient comparison exhibits the role of waves reflection in vapour behaviour during boiling. This phase of creation and oscillation of vapour slugs is followed by a maximum of vaporization estimated in the following subsection.

\subsection{Vapour created during the transient}

The previous subsection described the boiling onset. It clearly revealed that intense boiling generates a relatively large voiding along the tube before ascending the tube and reaching the vessel. The purpose of this section is to quantify the time variation of the vapour volume generated by boiling. The vapour behaves as follows:

(i) Boiling onset: creation of the first bubbles [at $t=0.0019 \mathrm{~s}$ ] (see figure 4 )

(ii) Boiling: motion of the vapour on the tungsten sheets [for $t=0.0019 \mathrm{~s}$ to $t \approx 0.048 \mathrm{~s}]$ (see Subsection 4.1)

(iii) Saturation of the viewport: the bubbles increase in size until the saturation of the viewport $\mathrm{V} 1$ [for $t=0.05 \mathrm{~s}$ ]

(iv) Departure of the vapour: the heap of vapour detaches itself from the tungsten sheets [for $t>0.05 \mathrm{~s}$ ]. It ascends in the tube as an oscillating vapour mass. Small bubbles lead a distinct large bubble and mediumsized bubbles trail comes after (see figure 7 at $t=0.45 \mathrm{~s}$ )

(v) Last bubble seen on V1 at $t \approx 0.35 \mathrm{~s}$ 
After identifying each phase, a post-processing on Matlab has been performed to compare the increase of pressure recorded by P3 and the boiling phenomena seen through V1. This post-processing consists in determining the visible area perturbed by the vapour bubble.

Figure 11 shows the Matlab process used with a benchmarked frame (a). The image used is from the viewport V2. The image (c) is the first frame (at $t=0 \mathrm{~s}$ ). From left to right, the first step is to compute the complement of the frame. It gives the resulting frames (b) and (d). Then by dividing both results the frame (e) is obtained. The final step is to use bwboundaries function from Matlab to distinguish and output all regions (f). The sum of the area of all the regions at each time step gives the visible vapour surface.

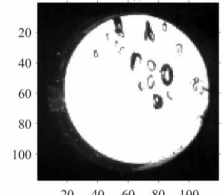

(a)

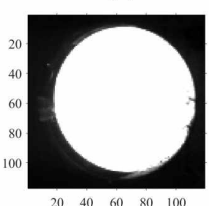

(c)

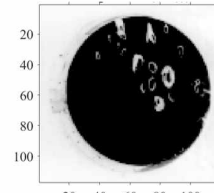

(b)

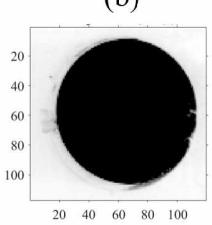

(d)

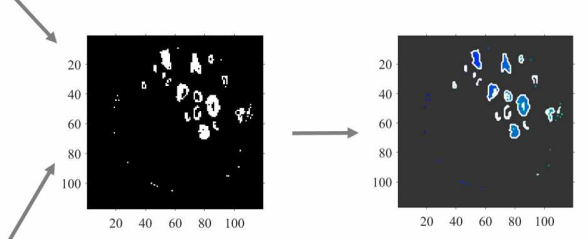

(e)

(f)

Figure 11: Frame processing performed in Matlab. (a) and (c) are the input of the calculus (respectively the considered and the initial frame). (f) is the outcome.

Figure 12 exhibits the vapour area calculated from viewport V1 (black curve) and the smoothed overpressure P3 (orange curve). The computed area displays three distinct phases: the increase surface of visible vapour (I), a plateau at $1500 \mathrm{px}^{2}$ (II), and a decrease of the visible surface of vapour (III). 


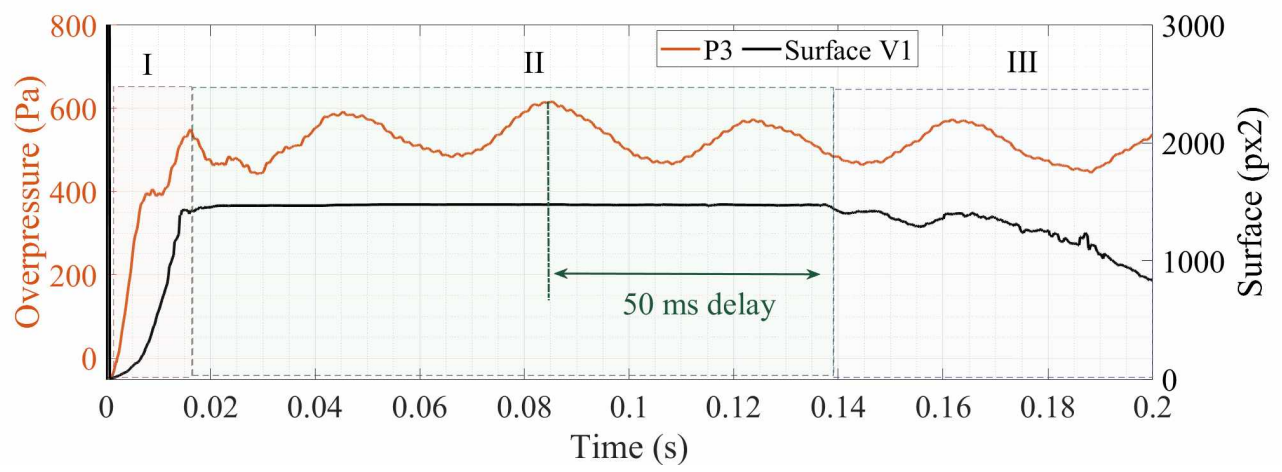

Figure 12: Vapour area seen on viewport V1 and compared to the overpressure compression in the buffer layer. I is the growth phase. II delimits the 'stable' stage. III stands for the decrease phase.

The vapour generated by boiling on the heating element leads to the increase of pressure within the test section. The vapour is generated under the buffer layer free surface (see figure 3). These changes of pressure are registered in the buffer layer by the sensor P3. As observed on figure 12, smoothed overpressure P3 exhibits three phases: a compression (increase of pressure between $0 \mathrm{~s}$ and $0.018 \mathrm{~s}$ ) then a fluctuating stabilization, between $0.02 \mathrm{~s}$ and $0.085 \mathrm{~s}$, and finally a (fluctuating) decrease starting near $0.085 \mathrm{~s}$. The vapour area and the overpressure P3 exhibits analogue behaviours:

I. Creation of vapour which implies an increase of pressure in the buffer layer (compression)

II. Saturation of the viewport V1 due to a maximum of vapour created

III. Departure of the vapour from the heating element which corresponds (with a $50 \mathrm{~ms}$ delay) to the decrease of overpressure.

It is in accordance with the visual chronology observed by watching the experiment movie and described at the beginning of the subsection (boiling enumeration).

These similar behaviours justify the use of P3 measurements to calculate the volume of vapour created $V_{v}$. The generated vapour has a mass $m_{v}$ and a volume $V_{v}=m_{v} / \rho_{v}$. The vapour generated will replace the volume occupied by the liquid and push the liquid upward. Under the assumption of a nearly incompressible liquid, the net expansion due to the vapour created 
is proportional to the vapour and the liquid density, respectively $\rho_{v}$ and $\rho_{l}$ as follows

$$
V_{n e t}=\left(1 / \rho_{v}-1 / \rho_{l}\right) m_{v}
$$

The volume of vapour created $V_{v}$ is calculated from the net compression as:

$$
V_{v}=V_{\text {net }} \frac{1}{1-\rho_{v} / \rho_{l}}
$$

Assuming the buffer layer transformation induced by the compression to be isentropic gives the Laplace coefficient expressed as follows

$$
\gamma=\frac{C_{P}^{(v)}}{C_{P}^{(v)}-\frac{z R}{M}}
$$

With, $C_{P}^{(v)}, R, T$ and $M$ the specific heat capacity of vapour at constant pressure $\left(\mathrm{J} . \mathrm{K}^{-1} \cdot \mathrm{kg}^{-1}\right)$, the gas constant $(8.314 \mathrm{~J} / \mathrm{K} / \mathrm{mol})$, the vapour temperature and the molar mass $\left(4410^{-3} \mathrm{~kg} / \mathrm{mol}\right)$ respectively. Carbon dioxide vapour at elevated pressure and near saturation can be satisfactorily described by a constant compressibility factor $z$ over the range of pressure and temperature fluctuations considered. At the initial thermodynamic state, $\left(P_{0}, T_{0}\right)=(2.96 \mathrm{MPa}, 266.8 \mathrm{~K}), z \approx 0.75$.

The isentropic compression of the buffer layer gives:

$$
P_{0} V_{0}^{\gamma}=\left(P_{0}+P_{3}\right)\left(V_{0}-V_{n e t}\right)^{\gamma}
$$

For the sake of clarity $V_{0}=V_{b l}(t=0)$, where the subscript $b l$ stands for 'buffer layer'. The volume of vapour created is predicted as follows:

$$
V_{v}=V_{0}\left(1-\left(\frac{P_{0}}{P_{0}+P_{3}}\right)^{1 / \gamma}\right) \frac{1}{1-\rho_{v} / \rho_{l}}
$$

Figure 13 shows the volume of vapour calculated thanks to equation (7). The curves of $\mathrm{P}_{3}$ and $V_{v}$ exhibits the same stages: vapour created (first $0.05 \mathrm{~s}$ ), maximum of vaporization reached (around $0.05 \mathrm{~s}$ ) and slow decrease (from $0.06 \mathrm{~s}$ ). The value of $V_{v}$ is fluctuating with a maximum nearly equal to $2.410^{-6} \mathrm{~m}^{3}$. This value is compared to the theoretical volume of vapour 
created. The theoretical volume is the ratio of the energy deposited with the latent heat of vaporization. It is calculated as $V_{v}^{(E l e c)}(t)=\left(\int U I d t\right) /\left(\mathcal{L}_{\rho_{v}}\right)$ and plotted on figure 13 (orange curve). The re-condensation of vapour is not considered in this calculation that therefore corresponds to a theoretical maximum vapour volume. It exhibits a second-order system's rise to a maximum value equal to $2.810^{-6} \mathrm{~m}^{3}$.

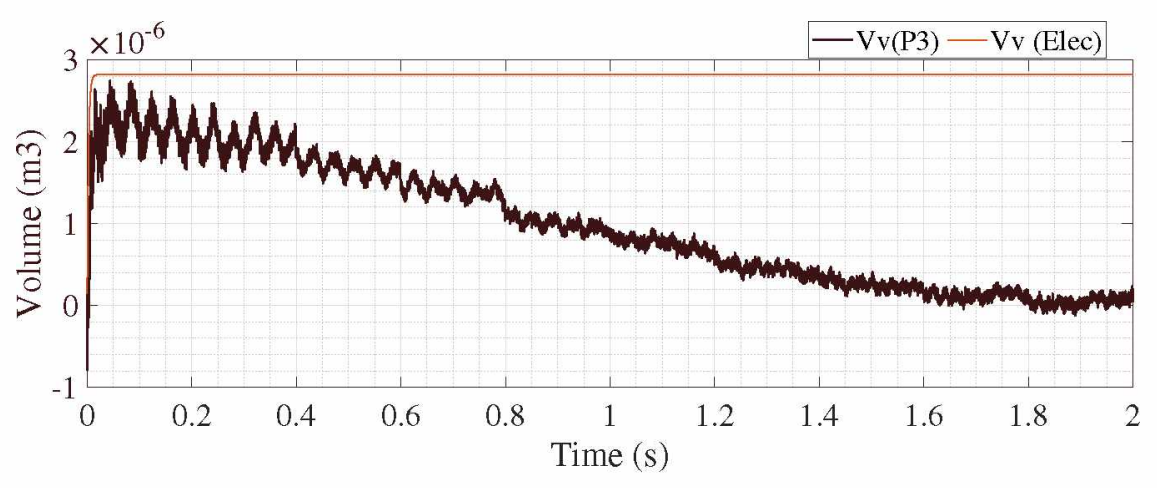

Figure 13: Volume of vapour created $(V v(P 3))$ compared to the theoretical volume of vapour created $(V v(E l e c))$

Both maxima are of the same order of magnitude and occurred at $0.05 \mathrm{~s}$ and $0.015 \mathrm{~s}$ respectively for $V_{v}$ and $V_{v}^{(\text {elec })}$. The theoretical rise time (from $\left.V_{v}^{(e l e c)}\right)$ is similar to the vapour surface rise time outputted on figure 12. This indicates that the main part of vapour is already generated at the end of the power discharge.

The rate of creation of vapour captured with the sensor P3 could lead to the characterization of rough analysis of the mean heat transfer on the tungsten plates. The current lack of relevant calculation of temperature of the tungsten during time is the main drag of thermal analysis on this experiment. Nevertheless, under the adiabatic assumption, the heating of the element would have led to a tungsten's temperature of approximately $285.2 \mathrm{~K}$. Its difference with the saturation temperature is named $\Delta T$ and is equal to $18.4 \mathrm{~K}$. Considering that all energy deposited in the fluid permits a phase change, a mean heat coefficient $h$ could be calculated from the volume of vapour created,

$$
h=\frac{\mathcal{L} \rho_{v} \frac{d V_{v}}{d t}}{S \Delta T}
$$


With $\mathcal{L}, \rho_{v}$ and $S$ are respectively the specific latent heat of liquid carbon dioxide $\left(2.4910^{5} \mathrm{~J} / \mathrm{kg}\right)$, the density of the vapour $\left(78.28 \mathrm{~kg} / \mathrm{m}^{3}\right)$ and the exchange surface between the heating element and the fluid $\left(2.9810^{-3} \mathrm{~m}^{2}\right)$. During the creation of vapour (phase I), the volume of vapour increase with a rate of $3.7010^{-4} \mathrm{~m}^{3} / \mathrm{s}$. It gives a mean maximum heat exchange equal to $1.2710^{5} \mathrm{~W} \cdot \mathrm{K}^{-1} \cdot \mathrm{m}^{-2}$. The mean (maximum) heat flux is then equal to 2.34 MW. $\mathrm{m}^{-2}$. This value is slightly higher than the one calculated in FCI experiments [7], $9.210^{4} \mathrm{~W} . \mathrm{m}^{-2}$ and $4.210^{5} \mathrm{~W} \cdot \mathrm{m}^{-2}$. But this value is smaller than the theoretical upper limit of heat flux, achieved by Schrage [Carey], $1.7510^{9} \mathrm{~W} \cdot \mathrm{m}^{-2}$.

Between $0.18 \mathrm{~s}$ and $2 \mathrm{~s}$, figure 13 reveals a relaxation of the buffer layer. During this period of time, the vapour has detached from the heating element, and ascends -first in the tube and then in the vessel. This ascend is seen on figure 7. The relaxation of the buffer layer is mainly due to the time constant of the piezoelectric sensors. However, the characterization of the bubbles brought information on the vapour behaviour during the ascend. The bubble dynamic during this phase is studied in the following subsection.

\subsection{Bubble departure and shape}

During the vapour ascends in front of V2 and V3, the velocity of the bubbles oscillate around a mean value. This suggests that they already reached their terminal velocity when passing through V2 and V3. It is then considered that the vapour ascends with an homogeneous velocity.

Figure 14 shows the motion of distinguishable bubbles situated in front of V3 from $t=0.42 \mathrm{~s}$ to $t=0.44 \mathrm{~s}$. To differentiate each time step, the jet colormap (Matlab colormap, [14]) is used. The blue colour is for the first frame (at $t=0.42 \mathrm{~s}-$ (b)) and the red colour for the last one (at $t=0.44 \mathrm{~s}-$ (c)). 


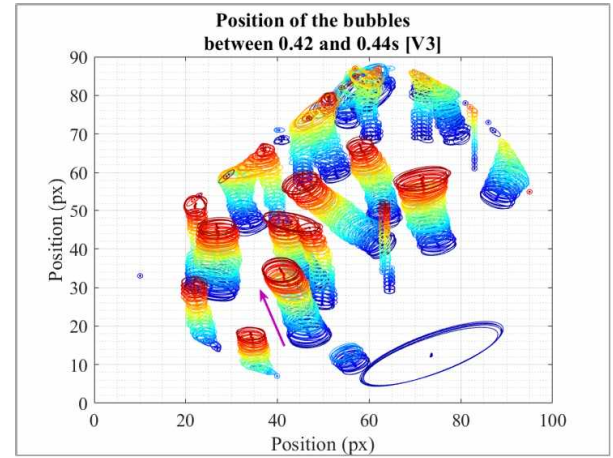

(a)

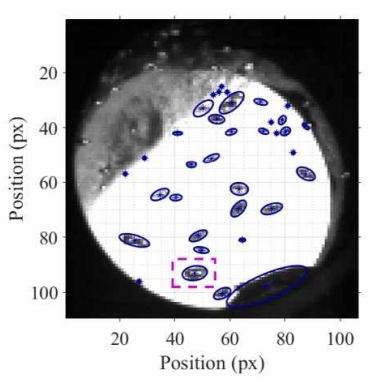

(b)

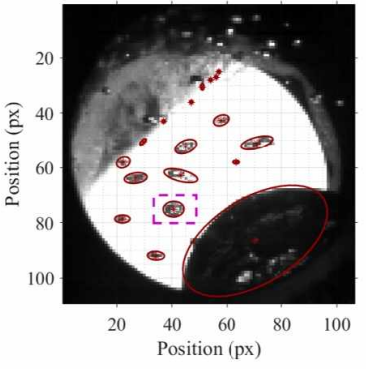

(c)

Figure 14: Tracking of one bubble in front of the viewport V3 (outlined in purple in each picture). The biggest bubble is suppressed (when its surface is too prominent) when plotting all the position (clearness matters)

To characterize the bubbly flow, the evolution of the shape and the velocity of the bubbles must be calculated. A well-defined and representative bubble is chosen and highlighted on figure 14 with a purple arrow (a) and dashed rectangle (b)-(c). To extract both information, an optical flow code based on classic-c-brightness method [15] is used. The use of Matlab functions and optical flow grants access to the velocity and the equivalent diameter of the particle at each time step. By overlapping each vapour particle by an ellipse, the equivalent diameter is calculated as follows:

$$
D_{e}=\sqrt[3]{8 a b^{2}}
$$

Where $a$ and $b$ are the minor and major axis length respectively.

The diagram of Grace, Weber and Clift is used to analyse the bubbly flow regime. It predicts the bubbly flow for given Eötvös ( $\left.E_{O}\right)$ and Reynolds (Re) numbers. For each time steps those numbers are calculated:

$$
\left\{\begin{array}{l}
\operatorname{Re}(t)=\left(\rho_{l} D_{e} u_{5}(t)\right) / \mu_{l} \\
E_{o}(t)=\left(g\left(\rho_{l}-\rho_{v}\right) D_{e}(t)^{2}\right) / \sigma
\end{array}\right.
$$

Introducing $\mu_{l}, g$ and $\sigma$ respectively the dynamic viscosity (Pa.s), the universal constant of gravitation $\left(9.81 \mathrm{~m} / \mathrm{s}^{2}\right)$ and the liquid surface tension $(\mathrm{N} / \mathrm{m})$. $u_{5}$ is the mean bubble velocity at each time $t$. This mean is calculated by averaging all the pixel velocities which constitute the bubble and averaging this value for 5 steps before $t$. The velocity is averaged over 5 time steps to 
avoid inconsistency due to the high camera frequency in regard to the real bubble velocity.

The diagram of Grace, Weber and Clift is shown on figure 15. It is divided in five different areas indicating the predicted bubble shape: spherical, ellipsoidal, skirted, spherical cap and wobbling. The previous calculation (see equation (10)) identifies the bubble selected as oscillating in shape and in velocity which confirms the camera observation.

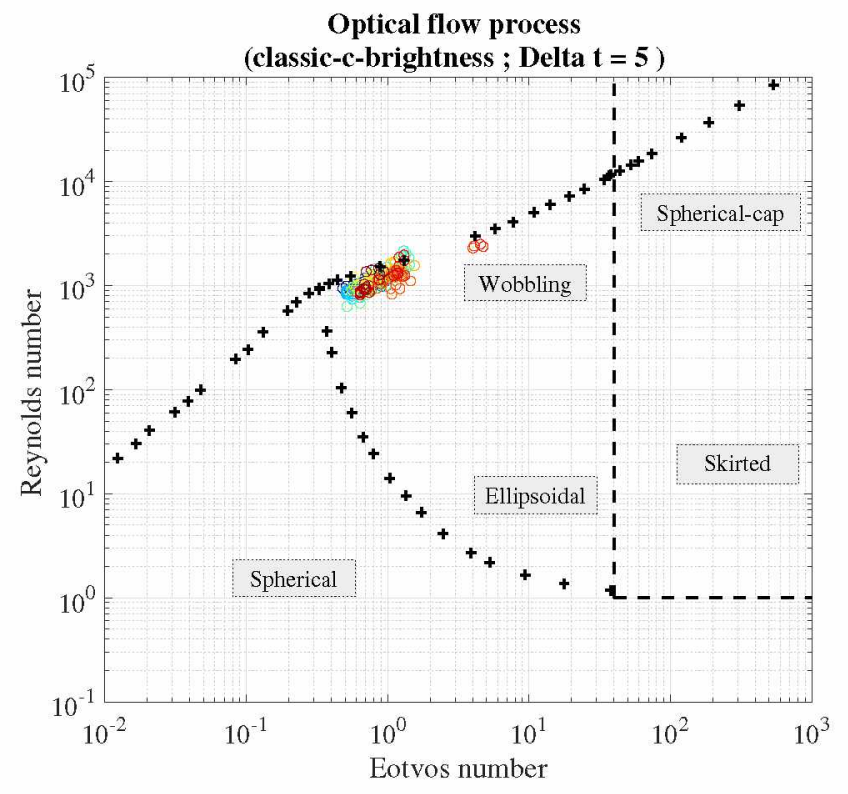

Figure 15: Bubble shape for the followed particle between pic $0.42 \mathrm{~s}$ and $0.44 \mathrm{~s}$

The mean equivalent diameter and the mean velocity $\left(u_{b}^{(e x p)}\right)$ of the considered bubble are respectively $1.2 \mathrm{~mm}$ and $0.145 \mathrm{~m} / \mathrm{s}$. By comparing this velocity to the literature (See [16]), the prediction of Clift and al. [17] is the nearest, giving $u_{b}^{(t h)}$ equal to $0.144 \mathrm{~m} / \mathrm{s}$. The formula giving this terminal velocity is:

$$
u_{b}^{(t h)}=\frac{\mu_{l}}{\rho_{l} \bar{D}_{e}} M o^{-0.149}(J-0.857)
$$

With, $\bar{D}_{e}$ the time averaged diameter (m), Mo $=\frac{g \mu_{l}^{4}\left(\rho_{l}-\rho_{v}\right)}{\rho_{l}^{2} \sigma^{3}}$ the Morton number and $J=38.56$. 
In conclusion to this ascending bubble study, we could expect wobbling regime for the bubble flow.

\section{Conclusion}

This study aims to present a first characterization of rapid boiling in saturated carbon dioxide. It is the first step to finely characterize vapour explosion taking place during a fuel coolant interaction (FCI). A dedicated experimental device based on Joule effect allows for a rapid deposition of energy into liquid $\mathrm{CO}_{2}$ confined in a tube part (where the heated plates lie) connected to a larger tank in the upper part. Rapid power release leads to a sharp pressure increase in the fluid tank as well as rapid and transient boiling, both being recorded by dedicated instrumentation.

The analysed test corresponds to a $55 \mathrm{~J}$ heating of a tungsten plate within $5 \mathrm{~ms}$. In $2 \mathrm{~ms}$ after the beginning of the energy deposition, a $1.310^{5} \mathrm{~Pa}$ overpressure peak is recorded near the heated plate. The increase rate of overpressure is equal to $163 \mathrm{MPa} / \mathrm{s}$ which is equivalent to the ones observed in BLEVE [1], but lower than the one observed in vapour explosion [10]-[9]. A pressure wave then propagates through the test section. Acoustic reflection and transmission at the cross section between the tube and the tank partially drive the pressure evolution. Rapid onset of boiling (few milliseconds after the energy deposit) can be seen on the plates. Bubble growth dynamics are affected by acoustic pressure wave and subsequent overpressure gradient variation. Most of the energy transmitted to the fluid is converted to latent heat and large voiding occurs around the heater at around $19 \mathrm{~ms}$ till vaporization process stops at $49 \mathrm{~ms}$. A rough heat transfer coefficient is calculated from the vapour production. It leads to mean maximum heat flux equal to $2.34 \mathrm{MW} \cdot \mathrm{m}^{-2}$. This value is slightly higher than the ones observed FCI experiments ([7]). However, since the test conditions are far from the ones encountered in nuclear power plant, any conclusion could be drawn from these values. Vapour detaches as a bubbly flow. Typical bubble size and velocity in the upward flow along the tube are respectively $1.2 \mathrm{~mm}$ (equivalent diameter) and $0.145 \mathrm{~m} \cdot \mathrm{s}^{-1}$. This order of magnitude is consistent with the prediction of Clift et al. [17] - [16].

Those results clearly indicate that the experimental device allows for the generation of the phenomenology associated with fuel coolant interaction in the context of nuclear safety scenarios as well as its characterization thanks to the dedicated instrumentation. The experimental results could then be 
used to validate models for the FCI. Latter work will correspond to the study of the variation of the phenomena with respect to control parameters of the transient, namely thermodynamic conditions (pressure, subcooling) and energy deposition (amplitude and time constant).

Very rapid boiling phenomena prove that the observed overpressure peak is related to the very first stages of the bubble growth under those highly transient heating conditions. Therefore, we consider improving the space and time resolution of the high-speed camera to better determine the time of first vaporization events along the plates. Some improvements concerning the online estimation of the volume occupied by the vapour could also help understanding the vaporization and condensation processes occurring within the device. A final improvement of the test section could be an online measurement of the power transmitted to the fluid; dedicated modifications of the experimental device are currently under development.

\section{Acknowledgements}

This work is funded by Électricité de France (EDF) and Institut de Radioprotection et de Sûreté Nucléaire in the frame of their collaborative research programs. The authors would deeply thank Laurent Zimmer from the CNRS and the Ecole Centrale Paris for his help on optical flow calculations. They would also thank the technical staff and the engineers who permit the mounting of the experimental test section in INSA Lyon, CETHIL.

\section{References}

[1] M. M. van der Voort, A. C. van den Berg, D. J. E. M. Roekaerts, M. Xie, P. C. J. de Bruijn, Blast from explosive evaporation of carbon dioxide: experiment, modeling and physics, Shock Waves 22 (2) (2012) 129-140. doi:10.1007/s00193-012-0356-0.

URL http://link. springer.com/10.1007/s00193-012-0356-0

[2] M. Xie, Thermodynamic and gasdynamic aspects of a boiling liquid expanding vapour explosion., Ph.D. thesis, Boxpress, 's-Hertogenbosch, iSBN: 9789088916748 OCLC: 905870250 (2013).

[3] N. Itoh, M. A. Sanchez, W.-C. Xu, K. Haraya, M. Hongo, Application of a membrane reactor system to thermal decomposition of CO2, Journal of Membrane Science 77 (2) (1993) 245-253. 
doi:10.1016/0376-7388(93)85073-6.

URL https://www.sciencedirect.com/science/article/pii/ 0376738893850736

[4] T. Qu, M. Zhou, 8 - Implosion of composite cylinders due to underwater impulsive loads, in: V. Lopresto, A. Langella, S. Abrate (Eds.), Dynamic Response and Failure of Composite Materials and Structures, Woodhead Publishing, 2017, pp. 239-262. doi: 10.1016/B978-0-08-100887-4.00008-1.

URL https://www.sciencedirect.com/science/article/pii/ B9780081008874000081

[5] G. Berthoud, Vapor Explosions, Annual Review of Fluid Mechanics 32 (1) (2000) 573-611, _eprint: https://doi.org/10.1146/annurev.fluid.32.1.573. doi:10.1146/ annurev.fluid.32.1.573.

URL https://doi.org/10.1146/annurev.fluid.32.1.573

[6] T. Fuketa, T. Fujishiro, Generation of destructive forces during fuel/coolant interactions under severe reactivity initiated accident conditions, Nuclear Engineering and Design 146 (1-3) (1994) 181-194. doi : 10.1016/0029-5493(94)90328-X.

URL https://linkinghub.elsevier.com/retrieve/pii/ 002954939490328X

[7] T. Sugiyama, T. Fuketa, Mechanical Energy Generation during High Burnup Fuel Failure under Reactivity Initiated Accident Conditions, Journal of Nuclear Science and Technology 37 (10) (2000) 877-886. doi: $10.1080 / 18811248.2000 .9714968$.

URL https://www.tandfonline.com/doi/full/10.1080/18811248. 2000.9714968

[8] T. Sugiyama, M. Umeda, T. Fuketa, H. Sasajima, Y. Udagawa, F. Nagase, Failure of high burnup fuels under reactivity-initiated accident conditions, Annals of Nuclear Energy 36 (3) (2009) 380-385. doi:10.1016/j. anucene.2008.12.003.

URL https://ww.sciencedirect.com/science/article/pii/ S0306454908003150 
[9] S. Glod, D. Poulikakos, Z. Zhao, G. Yadigaroglu, An investigation of microscale explosive vaporization of water on an ultrathin $\mathrm{Pt}$ wire, International Journal of Heat and Mass Transfer 45 (2) (2002) 367-379. doi:10.1016/S0017-9310(01)00158-2.

URL https://linkinghub.elsevier.com/retrieve/pii/ S0017931001001582

[10] Z. Zhao, S. Glod, D. Poulikakos, Pressure and power generation during explosive vaporization on a thin-(R) $l m$ microheater, Int. J. Heat Mass Transfer (2000) 16.

[11] A. Abbate, Etude expérimentale d'une interaction thermique au sein d'un fluide, Ph.D. thesis, Université de Lyon, INSA Lyon (2018).

[12] A. Kossolapov, F. Chavagnat, R. Nop, N. Dorville, B. Phillips, J. Buongiorno, M. Bucci, The boiling crisis of water under exponentially escalating heat inputs in subcooled flow boiling at atmospheric pressure, International Journal of Heat and Mass Transfer 160 (2020) 120137. doi:10.1016/j.ijheatmasstransfer.2020.120137.

URL https://ww.sciencedirect.com/science/article/pii/ S0017931020330738

[13] E. W. Lemmon, M. O. McLinden, D. G. Friend, National Institute of Standards and Technology (U.S.), Thermophysical properties of fluid systems, 1998, oCLC: 84549093.

URL http://webbook.nist.gov/chemistry

[14] Jet colormap array - MATLAB jet - MathWorks France. URL https://fr.mathworks.com/help/matlab/ref/jet.html

[15] D. Sun, S. Roth, M. J. Black, A Quantitative Analysis of Current Practices in Optical Flow Estimation and the Principles Behind Them, International Journal of Computer Vision 106 (2) (2014) 115-137. doi: 10.1007/s11263-013-0644-x. URL http://link. springer.com/10.1007/s11263-013-0644-x

[16] Y. Zhou, P. Kang, Z. Huang, P. Yan, J. Sun, J. Wang, Y. Yang, Experimental measurement and theoretical analysis on bubble dynamic behaviors in a gas-liquid bubble column, Chemical Engineering Science 211 (2020) 115295. doi:10.1016/j.ces.2019.115295. 
URL https://linkinghub.elsevier.com/retrieve/pii/ S0009250919307857

[17] R. Clift, J. Grace, M. Weber, Bubbles, Drops, and Particles, Dover Civil and Mechanical Engineering Series, Dover Publications, Incorporated, 2013. 